\title{
Role of Dehydroepiandrosterone Supplementation in Improving Intracytoplasmic Sperm Injection Outcome for Women with Expected Poor Ovarian Response
}

\author{
Abd El-Naser Abd El-Gaber Ali*, Mustafa M. Khodry \\ ART Unit of Obstetrics \& Gynecology Department, Qena Faculty of Medicine, South Valley University, Qena, Egypt \\ Email: *abdelnaser.abdelgaber@med.svu.edu.eg
}

How to cite this paper: Abd El-Gaber Ali, A.E.-N. and Khodry, M.M. (2019) Role of Dehydroepiandrosterone Supplementation in Improving Intracytoplasmic Sperm Injection Outcome for Women with Expected Poor Ovarian Response. Open Journal of Obstetrics and Gynecology, 9, 353-362.

https://doi.org/10.4236/ojog.2019.93036

Received: February 11, 2019

Accepted: March 5, 2019

Published: March 8, 2019

Copyright $\odot 2019$ by author(s) and Scientific Research Publishing Inc. This work is licensed under the Creative Commons Attribution International License (CC BY 4.0).

http://creativecommons.org/licenses/by/4.0/

\section{(c) (i) Open Access}

\begin{abstract}
Background: As regard to adjuvant supplementations, nowadays dehydroepiandrosterone (DHEA) is widely used all over the world and is considered to be a potential agent to ameliorate the assisted reproduction technologies outcomes of infertile women with poor ovarian reserve. Objective: To find out the role of DHEA supplementation in improving intracytoplasmic sperm injection (ICSI) outcome for infertile women with expected poor ovarian response in controlled ovarian stimulation. Setting: Assisted reproduction unit of Obstetrics and Gynecology Department, Faculty of Medicine, South Valley University, Egypt. Duration: From April 2016 to May 2018. Study Design: A randomized double-blinded controlled trial. Methods: One hundred and forty infertile women with expected poor ovarian response prepared for ICSI procedure were included in this study. Patients were divided into two groups; group I (DHEA group) included 70 patients received $25 \mathrm{mg}$ DHEA 12 weeks prior to ICSI cycle and group II (placebo group) included 70 patients received a placebo. Results: There was a highly statistically significant difference in basal AFC at start of ICSI cycle in group I (who received DHEA supplementation for 12 weeks prior to ICSI procedure) than in group II (13.8 \pm 5.3 versus $10.7 \pm 4.6$ respectively) with $\mathrm{P}<0.001$. There were mildly statistically significant differences between group I and group II as regard to increase in the number and quality of retrieved oocytes, increased in endometrial thickness, fertilization rate and embryo quality with $\mathrm{p}$ value $<0.05$ but there was no statistically significant difference between the 2 groups as regard to pregnancy (chemical and clinical) rates ( $\mathrm{p}$ value $>0.05$ ). Conclusions \& Recommendations: DHEA supplementations improved basal AFC, increased the number \& quality of oocytes and increased quality of embryos in infertile patients
\end{abstract}


with expected poor ovarian response in ICSI procedure. So DHEA supplementations could be an important adjuvant for infertile women with expected poor ovarian response in ICSI procedure.

\section{Keywords}

Dehydroepiandrosterone (DHEA), Intracytoplasmic Sperm Injection (ICSI), Poor Ovarian Response (POR)

\section{Introduction}

Assisted reproductive technology (ART) represented mainly in vitro fertilization (IVF) and intracytoplasmic sperm injection (ICSI) is considered the corner stone in solving infertility problem especially with global increase in obesity which has a negative impact on fertility and ovarian reserve [1]. Dehydroepiandrosterone (DHEA) is a multifunctional prohormone secreted from adrenal gland and acts as a precursor for both estradiol and testosterone synthesis [2] also has been reported to increase pregnancy and live birth rates especially in poor responders [3]. Besides dehydroepiandrosterone (DHEA) has been sought to improve fertilization rates and increase quality embryos [4] [5] [6] [7].

Dehydroepiandrosterone (DHEA) is considered one of the most common two adjuvant therapies in IVF and ICSI but due to lack of sufficient randomized controlled trials (RCTs) in this topic, the actual beneficial effects of adjuvant therapies especially dehydroepiandrosterone are debated and so far unclear [8]. Dehydroepiandrosterone was considered an active agent in improvement of IVF outcomes particularly in poor responders [9] [10]. High concentration of dehy dehydroepiandrosterone in the follicular fluid was associated with good ovarian response as regard to increase in the number of mature oocytes, besides it was associated with increase in (fertilization, implantation and live birth) rates [11]. Many studies reported that, the improving effect of dehydroepiandrosterone on IVF and ICSI outcomes could be explained as DHEA enhanced mitochondrial function and decreased the apoptosis of cumulus cells (CCs) [12] [13]. Ovarian reserve tests were classified into biochemical basal tests and provocative tests and ultrasound scanning of the ovaries on day 2 or 3 of menstrual cycle. Day-3 follicle-stimulating hormone (FSH) was the first test that was introduced in 1988, then clomiphene citrate challenge test (CCCT) in 1989, gonadotrophin releasing-hormone $(\mathrm{GnRH})$ agonist in 1989, inhibin B in 1997, antral follicular count (AFC) in 1997, and finally antimüllerian hormone (AMH) in 2002. Most of these tests have poor predictive values, often for their indirect way for ovarian reserve assessment (e.g. FSH, CCCT and GnRH agonist) also they have sometimes intracycle or intercycle variability (e.g. FSH) [14] [15]. The most reliable ovarian reserve markers used in clinical practice nowadays are basal AFC, and AMH [16]. 


\section{Aim of Work}

The aim of this study was to find out the role of dehydroepiandrosterone (DHEA) supplementation in improving ICSI outcome for infertile women with expected poor ovarian response.

\section{Methodology}

This study was conducted on 140 infertile women undergoing ICSI procedure with expected poor ovarian response (POR) according to presence of Bologna criteria, from ESHRE (European Society of Human Reproduction and Embryology) [1]. Infertile women had been defined as poor responders when they had at least two of the following three criteria; 1$)$ women with advanced age $(\geq 40)$ or the presence of any other risk factor for POR, 2) the presence of previous Poor Ovarian Response (when 3 or less oocytes retrieved with a conventional stimulation protocol), 3) presence of any abnormalities in Ovarian Reserve Tests (ORT) (as AFC (anteral follicle count) $<5-7$ or AMH $<0.5-1.1 \mathrm{ng} / \mathrm{mL}$ ).

Intracytoplasmic Sperm injection (ICSI) procedure was done at assisted reproduction unit of Qena university hospital, South Valley University, Egypt after complete infertility evaluation from April 2016 to May 2018. A verbal counseling and a clear written consent were obtained from all participants couples included in this study according to (The Committee of Faculty of Medicine Medical Ethics, South Valley University. Inclusion criteria; age (35 - 42) years, BMI $\leq 30$, Anti-mullerian hormone $(<1.0) \mathrm{ng} / \mathrm{dl})$. Exclusion criteria; presence of any gynecological problem (e.g. fibroid, endometriosis, uterine polyp, hydrosalpinx, adenomysis, previous ovarian cystectomy or oophorectomy for one ovary, history of cytotoxic chemotherapy or history of pelvic irradiation, abnormal sperm morphology (globozoospermia and pin point sperm), azoospermia; (whether obstructive or non-obstructive)). All cases included in this study subjected to the followings; 1) full history and clinical examination. 2) Ovarian reserve testing (serum AMH, basal serum FSH, basal AFC by transvaginal 2 dimensional ultrasound). 3) Uterine cavity assessment (by transvaginal 3-dimentional ultrasound or office hysteroscopy). 4) routine investigations ( $\mathrm{CBC}$, blood grouping and $\mathrm{Rh}$ typing, TSH, liver function tests, kidney function tests, prothrombin time, prothrombin concentration, blood sugar). 5) Semen analysis.

Methodology: Patients included in this study were classified into two groups; Group I (DHEA group $=70$ patients), received dehydroepiandrosterone (DHEA) $25 \mathrm{mg}$ twice daily 12 hours apart) and Group II (Placebo group $=50$ patients) received placebo (starch tablets) twice daily. Randomization was done in this study with closed envelops method. Providers and patients where blinded to the contents of the bags till the end of the study. DHEA had been administered in group I for 12 weeks prior to the ICSI procedure schedule and continued throughout the whole stimulation period till the HCG trigging time, and placebo (starch tablets) was received in group II twice daily till triggering time. All patients in both groups received combined contraceptive pills in menstrual 
cycle just prior to the ICSI cycle. In both groups gonadotrophins stimulation started at day 2 of the menstrual cycle using (Gonal F pen, a recombinant FSH, Serono, Aubonne, Switzerland) with hMG (Menogon, Ferring $75 \mathrm{iu}$ ) in 300 - 450 $\mathrm{iu}$ /day initiated dose, follicular monitoring was done by trans-vaginal ultrasound at the $6^{\text {th }}$ day of stimulation and then repeated every other day till two or more follicles on one or both ovaries achieved $14 \mathrm{~mm}$ or more in mean diameter, by that time gonadotrophin releasing hormone $(\mathrm{GnRH})$ antagonist (Cetrotide, Merck Seronoin $0.25 \mathrm{mg}$ ) which was daily injected subcutaneously until the triggering time. Triggering for final follicular maturation was done with using recombinant human chorionic gonadotrophins (HCG) (Ovitrelle $500 \mathrm{iu}$ ) that was injected subcutaneously. Cancellation of the cycle was considered if less than 2 follicles that achieved $18 \mathrm{~mm}$ or more in mean diameter. Oocytes were retrieved about 34 - 36 hours post triggering with HCG and intracytoplasmic sperm injection (ICSI) procedure was done for all patients in the two groups. Only $1-2 \mathrm{em}-$ bryos (day 3 or day 5) with (A\&B) qualities were allowed to be transferred.

\section{Outcome Measures}

Primary outcome; had assessment of basal antral follicle count (AFC), number and quality of retrieved oocytes, the total doses and duration of the gonadotrophins of stimulation, Estradiol levels, endometrial thickness and its pattern at time of ova pickup and number of cancelled cycles.

Secondary outcome; had assessed fertilization rate, number of good quality embryos and the pregnancy (chemical and clinical) rates. Serum $\beta$-hCG levels were assayed 14 days after embryo transfer and transvaginal ultrasound was done 21 days later for positive HCG cases (chemical pregnancy) to confirm clinical pregnancy by appearance of gestational sac or sacs, fetal viability detected by observing fetal heart valve movements. Luteal phase support was done with (prontogest, ABSA, ampoule, $100 \mathrm{mg}$ ) was intramuscular injected daily starting immediately after ova pickup and continued for two weeks post embryo transfer and extended till the end of first trimester in clinically pregnant cases.

\section{Statistical Analysis}

Analysis and statistical presentation of this study were conducted, using the mean, standard error, unpaired student t-test, linear correlation coefficient, Paired t-test and chi-square tests by using the Statistical Program for Social Sciences (SPSS Inc., Version 21.0, Chicago, IL, USA). A two sided $\mathrm{P}<0.05$ was considered to be a mildly statistically significant, $\mathrm{p}<0.01$ was considered to be moderately significant and $\mathrm{p}<0.001$ was considered highly significant.

\section{Results}

2 cases $(2.8 \%)$ in group I and 5 cases $(7.1 \%)$ in group II had cancelled ICSI cycle due to failure of follicular recruitment.

There were no statistically significant differences between group I and group II as regard to patient's basal characteristics with $\mathrm{p}$ value $>0.05$ (Table 1). There 
was a highly statistically significant difference in basal AFC at start of ICSI cycle in group I than in group II (13.8 \pm 5.3 versus $10.7 \pm 4.6$ respectively) with $\mathrm{P}<$ 0.001 (Table 2). There were mildly statistically significant differences between group I and group II as regard to increase in the number and quality of retrieved oocytes, increased in endometrial thickness, fertilization rate and embryo quality with $\mathrm{p}$ value $<0.05$ (Table 3, Figure $1 \&$ Figure 2 ) but there was no statistically significant difference between the 2 groups as regard to the duration of stimulation (days), the dose of gonadotrophins (IU) and in the pregnancy (chemical and clinical) rates with $\mathrm{p}$ value $>0.05$ (Table 3 and Figure 3 ).

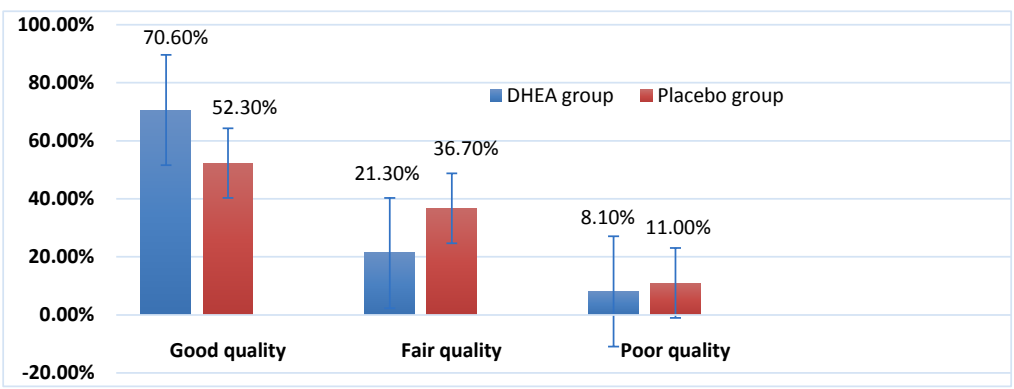

Figure 1. Comparison between group I and group II as regard to oocyte quality. Mildly significant ( $\mathrm{p}$ value $<0.05$ ).

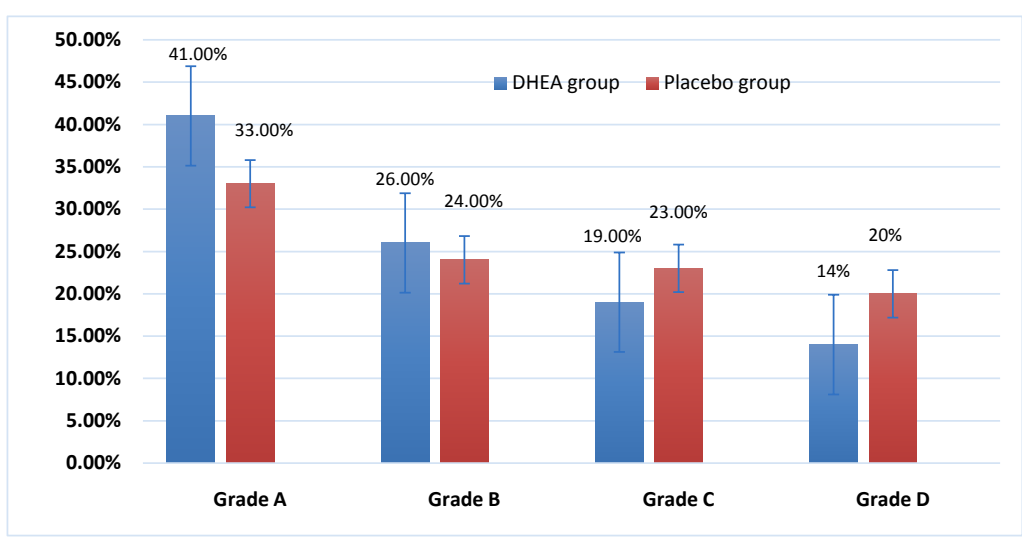

Figure 2. Comparison between group I and II as regard to embryo quality. Mildly significant ( $\mathrm{p}$ value $<0.05$ ).

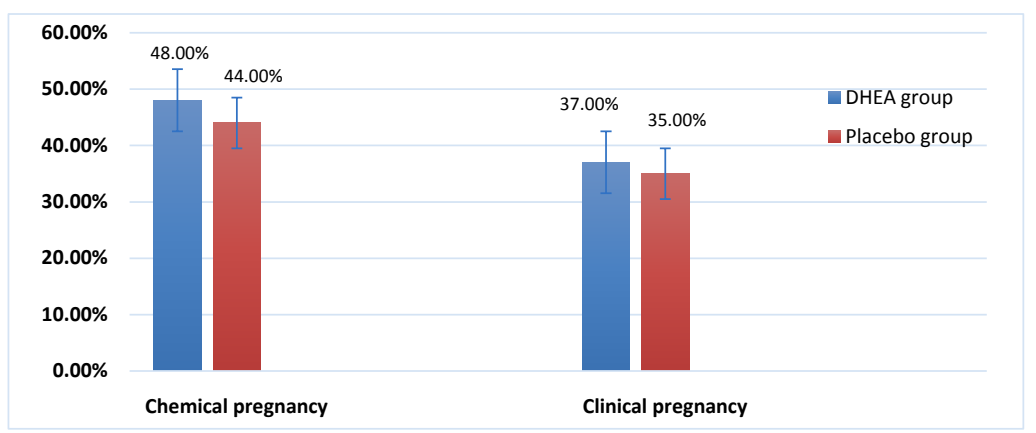

Figure 3. Incidence of pregnancy rates in group I and group II. Non-significant $(\mathrm{P}$ value $>0.05)$. 
Table 1. Baseline characteristics in Group I and Group II.

\begin{tabular}{cccc}
\hline & $\begin{array}{c}\text { GI (DHEA) } \\
\mathbf{N}=70\end{array}$ & $\begin{array}{c}\text { GII (Placebo) } \\
\mathbf{N}=70\end{array}$ & P-value \\
\hline Age (years) mean \pm SD & $38.5 \pm 5.6$ & $37.0 \pm 4.08$ & NS \\
BMI $\left(\mathrm{kg} / \mathrm{m}^{2}\right)$ mean $\pm \mathrm{SD}$ & $27.1 \pm 3.4$ & $27.4 \pm 2.7$ & $\mathrm{NS}$ \\
Duration of infertility (years) mean $\pm \mathrm{SD}$ & $6.9 \pm 4.0$ & $7.4 \pm 3.6$ & $\mathrm{NS}$ \\
AMH (ng/dl) mean \pm SD & $0.8 \pm 0 . .2$ & $0.9 \pm 0.3$ & NS \\
Basal AFC mean $\pm \mathrm{SD}$ & $3.2 \pm 1.1$ & $3.4 \pm 1.3$ & NS \\
\hline
\end{tabular}

NS $=$ non-significant $(\mathrm{p}$ value $>0.05)$

Table 2. Comparison between Group I and Group II as regard to basal antral follicle count (AFC) at start ofintracytoplasmic sperm injection (ICSI) cycle.

\begin{tabular}{lccc}
\hline & $\begin{array}{c}\text { GI (DHEA) } \\
\mathbf{N}=\mathbf{7 0}\end{array}$ & $\begin{array}{c}\text { GII (Placebo) } \\
\mathbf{N}=\mathbf{7 0}\end{array}$ & P-value \\
\hline AFC at start of ICSI cycle mean \pm SD & $13.8 \pm 5.3$ & $10.7 \pm 4.6$ & HS \\
\hline
\end{tabular}

HS $=$ highly significant $(\mathrm{p}$ value $<0.001)$.

Table 3. Comparison between group I group II as regarding total doses of gonadotrophins, duration of stimulation, in number of oocytes retrieved, endometrial thickness and fertilization rate.

\begin{tabular}{cccc}
\hline & $\begin{array}{c}\text { GI (DHEA) } \\
\mathrm{N}=68\end{array}$ & $\begin{array}{c}\text { GII (Placebo) } \\
\mathrm{N}=65\end{array}$ & P-value \\
\hline Duration of stimulation (days) mean \pm SD & $13.2 \pm 1.5$ & $13.8 \pm 1.7$ & $\mathrm{NS}$ \\
Dose of gonadotrophin (IU) mean \pm SD & $4882.1 \pm 1391.9$ & $4970.1 \pm 1554.2$ & $\mathrm{NS}$ \\
Number of retrieved oocytes mean $\pm \mathrm{SD}$ & $6.3 \pm 1.4$ & $3.9 \pm 1.2$ & $\mathrm{~S}$ \\
Endometrial thickness in mm mean $\pm \mathrm{SD}$ & $8.4 \pm 1.6$ & $6.6 \pm 1.4$ & $\mathrm{~S}$ \\
$\quad$ Fertilization rate (\%) & $76.4 \%$ & $60.7 \%$ & $\mathrm{~S}$ \\
\hline
\end{tabular}

$\mathrm{NS}=$ non-significant $(\mathrm{p}$ value $>0.05)-\mathrm{S}=$ mildly significant $(\mathrm{p}$ value $<0.05)$.

\section{Discussion}

Androgens have been proved in promotion the recruitment and in initiation the growth of primordial follicles and in the significant increase in number of primordial, pre-antral and antral follicles by up-regulation of IGF-1 [17]. Also androgens have a role in up-regulation of FSH receptor expression inside granulosa cells that could potentiate the FSH action [18] and to play a role in paracrine regulation in final maturation of the follicles and also minimize follicular atresia [19].

Dehydroepiandrosterone (DHEA) also has a role in decline incidence of aneuploidy in embryos that can be explained by induction of good quality oocytes [20]. Many studies has reported the benefits of DHEA in poor responders, but In 2000, Casson et al. [3] was the first who reported that DHEA could be used as adjuvant supplementation in poor responders may lead to an enhance- 
ment in ovarian response so in this study we tried to emphasize that effect on infertile women underwent ICSI procedure and expected to have poor ovarian response. The patients' characteristics were insignificant between DHEA group and Placebo group in our study as regard to age, BMI, duration, AMH and basal AFC with $p$ value $>0.05$ (Table 1 ). The AFC had a highly statistically significant increase in DHEA group after 12 weeks of DHEA supplementation (Table 2). Also this study reported that the number and quality of oocytes in DHEA group was increased with mildly statistically significant differences with ( $\mathrm{p}$ value $=$ 0.05 ) (Table 3 \& Figure 1). However there was no significant difference in gonadotrophin doses (IU) or in the duration of stimulation (Table 3).

In other hand this study had showed that embryos quality in DHEA group had a mildly significant difference in relation to placebo group with $\mathrm{p}$ value $<$ 0.05 (Figure 2) but as regard to pregnancy rates, were slightly high (both chemical and clinical) in DHEA group but still had no statistically significant difference with $p$ value $>0.05$ (Figure 3 ) and this can be explained the effect of DHEA supplementation 12 weeks prior to ICSI cycles increased oocytes number and qualities so enhanced fertilization rates and increased the percentages of good quality embryos in group I that enhanced embryo implantation so it had contributed in slight increase in pregnancy rates.

Many studies in literature had been discussed the beneficial effect of DHEA administration prior to ICSI or IVF cycles in poor responders and reported wonderful results as DHEA was very effective as supplementation for enhancement basal follicular count, oocyte number and quality, and may had a positive effect on embryo quality with or without increase in pregnancy rates, from these studies, Kuan-Hao et al. [21] study showed that antral follicle count was significantly increased, from $2.8 \pm 1.0$ before DHEA supplementation to $4.1 \pm 1.2$, and an increase in numbers of retrieved oocytes (from $2.4 \pm 1.1$ before DHEA supplementation to $4.2 \pm 1.2$; p < 0.01), also increased fertilized ova $(1.7 \pm 0.5$ increased to $3.8 \pm 1.1 ; \mathrm{p}<0.001$ ), Day 3 embryos increased (from $1.7 \pm 0.5$ before supplementation to $3.7 \pm 1.1 ; \mathrm{p}<0.001)$ and transferred embryos had increased (from $1.7 \pm 0.8$ before treatment to $2.8 \pm 0.8 ; \mathrm{p}<0.01$ )]. Also Hyman et al. [22] reported a significant increase in AFC $(5.3 \pm 2.8$ with DHEA versus $3.6 \pm 1.5$ in placebos; $\mathrm{p}<0.001$ ). The results of this study showed an improvement in basal AFC, oocyte number, quality, fertilization rate and embryo quality that may encourage assisted reproductive centers to use DHEA in infertile patients with expected poor ovarian response to improve the results and success rate of ICSI and IVF cycles so save time and decrease cost of repeated cycles. As regard to the optimal period of DHEA supplementation prior to ICSI or IVF cycles, different protocols has been reported in literature as 2, 3 or 4 months prior to ART cycles, Barad et al. [20] reported DHEA administration for more than months prior to ICSI which had increased cumulative pregnancy success rate, Gleicher et al. [20] reported more than 2 months of DHEA administration had a significant reduction in rate of miscarriage and Gleicher et al. [4] reported 1 - 3 months of DHEA supplementation had a significant reduction in embryos with aneuploidy, finally 
Fusi et al. [24] used DHEA more than 3 months in infertile women and had an increase in spontaneous pregnancy rate.

Limitation of this study was related to the small sample size (because many patients refused to be involved in the study) and applied only for ICSI procedure so further studies including large samples size in all varieties of assisted reproduction technology as IVF and IUI should be done to verify the exact benefits of DHEA supplementation in infertile women with expected poor ovarian.

\section{Conclusion \& Recommendation}

Dehydroepiandrosterone (DHEA) supplementations improved basal AFC, increased the number \& quality of oocytes and increased quality of embryos in infertile patients with expected poor ovarian response in ICSI procedure. So this study suggested DHEA supplementations as an effective adjuvant for infertile women with expected poor ovarian response in next ICSI procedure.

\section{Conflicts of Interest}

The authors declare no conflicts of interest regarding the publication of this paper.

\section{References}

[1] Ferraretti, A.P., La Marca, A., Fauser, B.C., Tarlatzis, B., Nargund, G., Gianaroli, L., et al. (2011) ESHRE Consensus on the Definition of 'Poor Response' to Ovarian Stimulation for In Vitro Fertilization: The Bologna Criteria. Human Reproduction, 26, 1616-1624. https://doi.org/10.1093/humrep/der092

[2] Arlt, W. (2004) Dehydroepiandrosterone and Ageing. Best Practice \& Research Clinical Endocrinology \& Metabolism, 18, 363-380. https://doi.org/10.1016/j.beem.2004.02.006

[3] Casson, P.R., Lindsay, M.S., Pisarska, M.D., Carson, S.A. and Buster, J.E. (2000) Dehydroepiandrosterone Supplementation Augments Ovarian Stimulation in Poor Responders: A Case Series. Human Reproduction, 15, 2129-2132. https://doi.org/10.1093/humrep/15.10.2129

[4] Gleicher, N., Weghofer, A. and Barad, D. (2010) Dehydroepiandrosterone (DHEA) Reduces Embryo Aneuploidy: Direct Evidence from Preimplantation Genetic Screening (PGS). Reproductive Biology and Endocrinology, 8, 140. https://doi.org/10.1186/1477-7827-8-140

[5] Kotb, M.M.M., Hassan, A.M.A. and Awadallah, A.M.A. (2016) Does Dehydroepiandrosterone Improve Pregnancy Rate in Women Undergoing IVF/ICSI with Expected Poor Ovarian Response According to the Bologna Criteria? A Randomized Controlled Trial. European Journal of Obstetrics \& Gynecology and Reproductive Biology, 200, 11-15. https://doi.org/10.1016/j.ejogrb.2016.02.009

[6] Barad, D. and Gleicher, N. (2006) Effect of Dehydroepiandrosterone on Oocyte and Embryo Yields, Embryo Grade and Cell Number in IVF. Human Reproduction, 21, 2845-2849. https://doi.org/10.1093/humrep/del254

[7] Zhang, H.H., Xu, P.Y., Wu, J., Zou, W.W., Xu, X.M., Cao, X.Y., et al. (2014) Dehydroepiandrosterone Improves Follicular Fluid Bone Morphogenetic Protein-15 and Accumulated Embryo Score of Infertility Patients with Diminished Ovarian Reserve 
Undergoing In Vitro Fertilization: A Randomized Controlled Trial. Journal of Ovarian Research, 7, 93. https://doi.org/10.1186/s13048-014-0093-3

[8] Nardo, L.G., El-Toukhy, T., Stewart, J., Balen, A.H. and Potdar, N. (2015) British Fertility Society Policy and Practice Committee: Adjuvants in IVF: Evidence for Good Clinical Practice. Human Fertility, 18, 2-15.

https://doi.org/10.3109/14647273.2015.985454

[9] Lin, L.T., Tsui, K.H. and Wang, P.H. (2015) Clinical Application of Dehydroepiandrosterone in Reproduction: A Review of the Evidence. Journal of the Chinese Medical Association: JCMA, 78, 446-453. https://doi.org/10.1016/j.jcma.2014.12.008

[10] Zhang, J., Qiu, X., Gui, Y., Xu, Y., Li, D. and Wang, L. (2016) Dehydroepiandrosterone Improves the Ovarian Reserve of Women with Diminished Ovarian Reserve and Is a Potential Regulator of the Immune Response in the Ovaries. Bioscience Trends, 9, 350-359. https://doi.org/10.5582/bst.2015.01154

[11] Chimote, N.M., Nath, N.M., Chimote, N.N. and Chimote, B.N. (2015) Follicular Fluid Dehydroepiandrosterone Sulfate Is a Credible Marker of Oocyte Maturity and Pregnancy outcome in Conventional In Vitro Fertilization Cycles. Journal of Human Reproductive Sciences, 8, 209-213. https://doi.org/10.4103/0974-1208.170397

[12] Tsui, K.H., Lin, L.T., Horng, H.C., Chang, R., Huang, B.S., Cheng, J.T., et al. (2014) Gene Expression of Cumulus Cells in Women with Poor Ovarian Response after Dehydroepiandrosterone Supplementation. Taiwanese Journal of Obstetrics and Gynecology, 53, 559-565. https://doi.org/10.1016/j.tjog.2014.09.003

[13] Lin, L.T., Wang, P.H., Chen, S.N., Li, C.J., Wen, Z.H., Cheng, J.T., et al. (2017) Protection of Cumulus Cells Following Dehydroepiandrosterone Supplementation. Gynecological Endocrinology: The Official Journal of the International Society of Gynecological Endocrinology, 33, 100-104. https://doi.org/10.1080/09513590.2016.1214262

[14] Scott Jr, R.T., Hofmann, G.E., Oehninger, S. and Muasher, S.J. (1990) Intercycle Variability of Day 3 Follicle Stimulating Hormone Levels and Its Effect on Stimulation Quality in In Vitro Fertilization. Fertility and Sterility, 54, 297-302. https://doi.org/10.1016/S0015-0282(16)53707-8

[15] Jayaprakasan, K., Campbell, B., Hopkisson, J., Clewes, J., Johnson, I. and Raine Fenning N. (2008) Establishing the Intercycle Variability of Three-Dimensional Ultrasonographic Predictors of Ovarian Reserve. Fertility and Sterility, 90, 2126-2132. https://doi.org/10.1016/j.fertnstert.2007.10.028

[16] Sharara, F.I., Scott, R.T. and Seifer, D.B. (1998) The Detection of Diminished Ovarian Reserve in Infertile Women. American Journal of Obstetrics \& Gynecology, 179, 804-812. https://doi.org/10.1016/S0002-9378(98)70087-0

[17] Vendola, K., Zhou, J., Wang, J. and Bondy, C.A. (1999) Androgens Promote Insulin-Like Growth Factor-I and Insulin-Like Growth Factor-I Receptor Gene Expression in the Primate Ovary. Human Reproduction, 14, 2328-2332. https://doi.org/10.1093/humrep/14.9.2328

[18] Nielsen, M., Rasmussen, I., Kristensen, S., Christensen, S., Møllgård, K., Andersen, E.W., et al. (2011) In Human Granulosa Cells from Small Antral Follicles, Androgen Receptor mRNA and Androgen Levels in Follicular Fluid Correlate with FSH Receptor mRNA. Molecular Human Reproduction, 17, 63-70. https://doi.org/10.1093/molehr/gaq073

[19] Hillier, S.G. and Tetsuka, M. (1997) Role of Androgens in Follicle Maturation and Atresia. Baillière's Clinical Obstetrics and Gynaecology, 11, 249-260. https://doi.org/10.1016/S0950-3552(97)80036-3 
[20] Gleicher, N., Ryan, E., Weghofer, A., Blanco-Mejia, S. and Barad, D.H. (2009) Miscarriage Rates after Dehydroepiandrosterone (DHEA) Supplementation in Women with Diminished Ovarian Reserve: A Case Control Study. Reproductive Biology and Endocrinology, 7, 108. https://doi.org/10.1186/1477-7827-7-108

[21] Tsui, K.-H., Lin, L.-T., Chang, R., Huang, B.-S., Cheng, J.-T. and Wang, P.-H. (2015) Effects of Dehydroepiandrosterone Supplementation on Women with Poor Ovarian Response: A Preliminary Report and Review. Taiwanese Journal of Obstetrics \& Gynecology, 54, 131e136.

[22] Hyman, J.H., Margalioth, E.J., Rabinowitz, R., Tsafrir, A., Gal, M., Alerhand, S., et al. (2013) Aprospective Study on Role of Dehydroepiandrosterone (DHEA) on Improving the Ovarian Reserve Markers in Infertile Patients with Poor Ovarian Reserve. Gynecological Endocrinology, 29, 989-992.

https://doi.org/10.3109/09513590.2013.824957

[23] Barad, D., Brill, H. and Gleicher, N. (2007) Update on the Use of Dehydroepiandrosterone Supplementation among Women with Diminished Ovarian Function. Journal of Assisted Reproduction and Genetics, 24, 629-634. https://doi.org/10.1007/s10815-007-9178-x

[24] Fusi, F.M., Ferrario, M., Bosisio, C., Arnoldi, M. and Zanga, L. (2013) DHEA Supplementation Positively Affects Spontaneous Pregnancies in Women with Diminished Ovarian Function. Gynecological Endocrinology, 29, 940-943.

https://doi.org/10.3109/09513590.2013.819087 